\title{
A BIRD'S-EYE VIEW
}

A falcon held between two hands. The bird of prey does not seem to be bothered by the human hand's touch, which reveals a kind of hole on the side of her head. It is her ear. The eyes, one of the falcon's most powerful instruments, are covered by a kind of white veil (fig. 1, plate I). She does not see as she is anaesthetised whilst her ear is "uncovered" by one of the numerous members of staff working at the Abu Dhabi Falcon Hospital. The United Arab Emirates on the Arabian Gulf belongs to a region whose heritage, and especially visual heritage, is based, among other things, on falconry, which is truly an ars vivendi.

Visual Engagements. Image Practices and Falconry focuses on intersections between culture and nature, vision and the gaze, tactility and perception, perspective and surveillance and questions concerning political iconology, as well as the migration of objects and images. However, epistemic notions around flying or cartography are also addressed.

Any practice is blind without a theory, but theories which do not consciously rely on practices are often problematic. It is of great importance to address the implicit knowledge involved in the pursuit of falconry as is the case with the image in general.

The present studies are not based on practical experience (none of the contributors is a falconer) but rather originate from a theoretical point of view that addresses the symbolic and epistemological level of the very practice. For the first time, theoretical reflection brings together two quite different areas in a non-systematic dialogue that evolves in various directions following different associations.

Considerations on the conditions and possibilities of perception in humananimal relationships through visual media and technology is certainly of primordial concern for research. The relationship between falcon and falconer, seen both through a cultural historical lens and by drawing on recent cognitive research on empathy, touches upon basic aspects of human-animal interactions.

Exploration of the faculty of seeing and the respective visual practices also has the result of stressing relationships between seeing and the handling of hawks in terms 


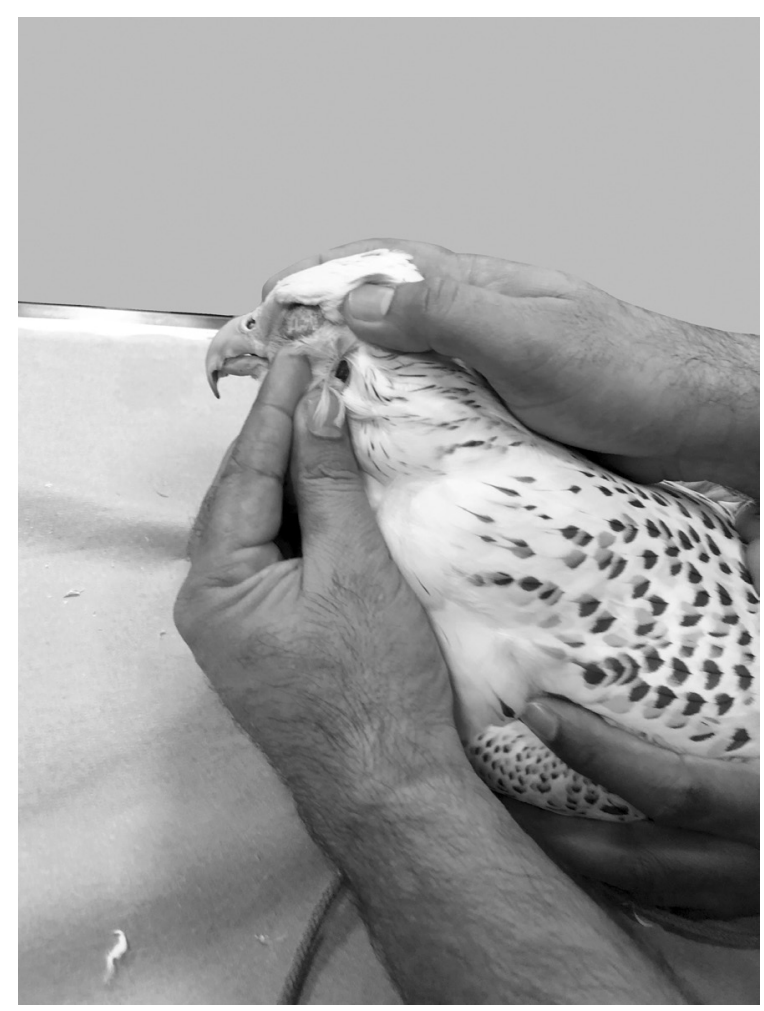

1 _. Anaesthetised falcon, Abu Dhabi, Falcon Hospital.

of perception. In more concrete terms the material furniture of falconry such as the hood and the lure, with the feathers that adorn them, bring together practical falconry with image practices, since the falconer is dependent on visual tactile devices that also have deeply symbolic connotations.

The faculty of seeing brings a metaphorical dimension to the topos of the gaze as an arrow, having a parallel in the relationship between image and observer. This analogy is also pertinent to relationships between the agency of the image and that of the hawk.

Falconry evokes a metaphor for surveying and cartographic gazing. It is a prosthesis of the human eye and body, involving touching and seeing. Here one has only to think of the so-called bird's-eye view that is a pictorial invention between perspective and measurement, a monitoring as a kind of organic drone avant la lettre. In this sense, visual engagements have a multisensorial dimension that one can combine not only with theories on empathy but also embodiment and enactive perception.

In a broader sense, of course, falconry is inextricably linked with flight and, as such, involves the human imagination. Aby Warburg worked exactly on this subject by coining the term "image vehicle" (Bilderfahrzeug). Beyond their actual movement image 


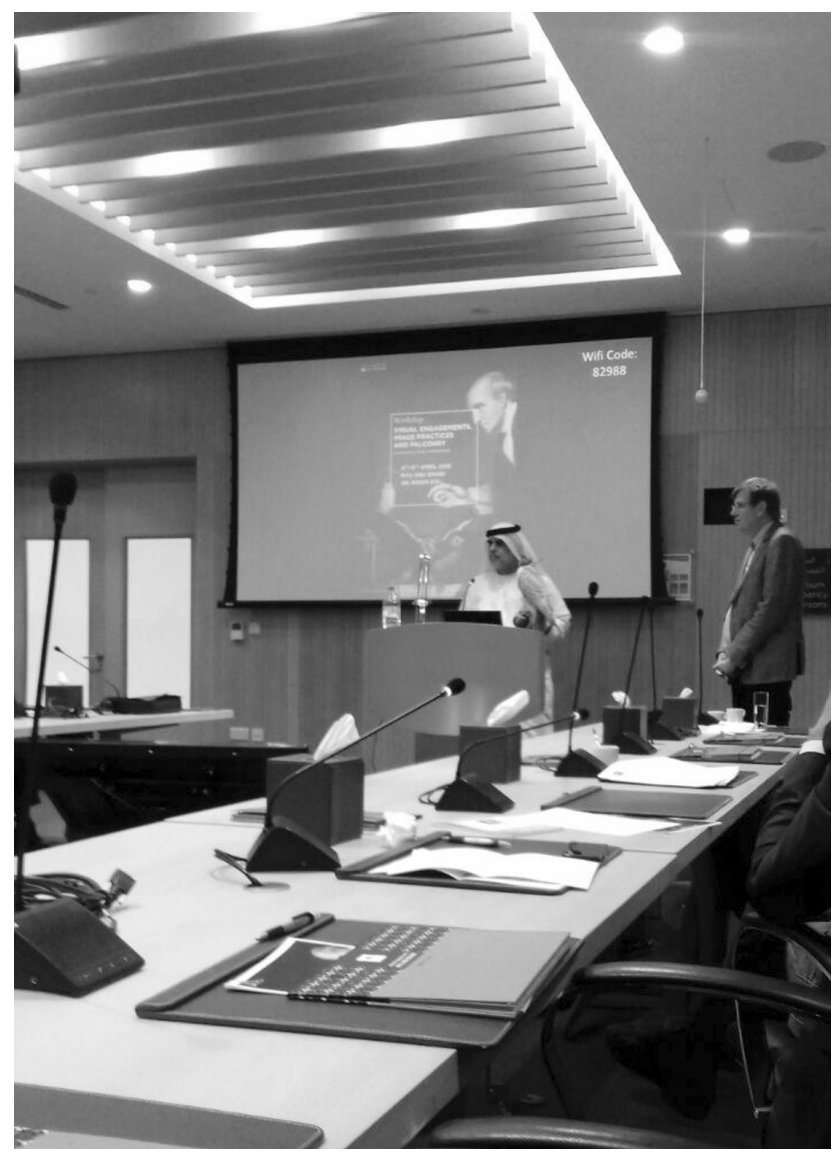

2 __ Falconer Mohammed al Hammadi during the workshop together with Gregor Stemmrich, Abu Dhabi, New York University.

vehicles can be metaphorical instruments of reflection on symbolic representation. However, there is also an epistemological notion of flying being a meeting point between natural laws and artistic imagination, blending both together to result in a rather paradoxical constellation of order and its negation, of possibility and impossibility. This consideration could serve as an emblem for the present endeavour, namely to prompt these different areas of comparison (falconry, visual imagery, the gaze or image vehicles) as a new terrain for research, questioning about their differences and, most of all, their similarities.

The aforementioned image (fig. 1) was taken during a visit on the first day of the workshop that took place in April 2018 at New York University, Abu Dhabi, within the framework of a research fellowship in the Humanities. I wish to especially thank 
Reindert Falkenburg, Martin Klimke and, last but not least, Alexandra Sandu for making things as easy as possible during my inspiring sojourn. Gila Wells and Nora Yousif also helped at different stages of the endeavour. The book was funded generously from the NYUAD institute grants as well as the Humanities Fellowship programme.

Andreas Beyer and Reindert Falkenburg also contributed, with their papers and their interventions at the overall discussions during the workshop. Gregor Stemmrich kindly chaired some of the sessions. I wish also to thank Emirati falconer Mohammed al Hammadi (Emirates Falconers' Club), who brought his falcon to NYUAD and discussed with us various questions concerning falconry training and manning processes (fig. 2). This was truly a moment when theory and practice indeed met in a fruitful interaction.

I would like to thank Baudouin van den Abeele and Horst Bredekamp who, although not present at the meeting in Abu Dhabi, contributed to the volume.

The publishing house De Gruyter expressed interest in undertaking this edition and I wish to particularly thank Susanne Drexler and Katja Richter for this, as well as Petra Florath for her imaginative work on the design and layout.

David Horobin of the British Archives of Falconry corrected all texts in English with great enthusiasm, enriching all of the papers with his comments and questions from the perspective of a practical falconer as well as that of a scholar.

The authors must be warmly thanked for their engaging contributions and for their stimulating presence during the workshop at NYU Abu Dhabi.

Y.H. 